\title{
PHYSICS AND THE WAVE EQUATION
}

\author{
J. C. SLATER
}

Often in the history of physics a guiding line of mathematical thought has permeated the whole of the science for years, tying together apparently unrelated branches of the subject, contributing to the unity of physics, but at the same time stimulating philosophical thought, and focussing attention on a branch of mathematics, and leading to its development. The Newtonian mechanics was such a guiding principle. From Kepler through Newton and up into the nineteenth century, more and more of physics was explicable in mechanical form. Even philosophy and economics and history felt the impact of rationalism. Mathematics felt the tide; calculus and the theory of ordinary differential equations grew up under the impetus of the physicist, who needed the mathematical methods to explain his physical facts. A second guiding principle was the variation principle. D'Alembert, Lagrange, Hamilton expressed the laws of mechanics in variational form. As time went on, more and more branches of physics could be formulated in similar language. We had not merely the principle of least action in mechanics, but Fermat's principle in optics, and variational formulations of electromagnetic theory. Here again there were impacts on both philosophy and mathematics. The philosophers grasped at the principle of least action as a proof of the existence of the deity, who used the simplest and most effective means to accomplish his purposes. The mathematicians were led to the development of the calculus of variations, and to such related fields as the theory of continuous groups and of contact transformations. Several similar developments have come since that time; two conspicuous ones are statistics, as seen in statistical mechanics, in the philosophical ideas associated with the second law of thermodynamics, and in the mathematical development of the theory of statistics; and relativity, with its obvious philosophical accompaniments, and its relation to the theories of the absolute differential calculus and tensor analysis. In all of these cases, I believe one could make out a case for the thesis that each succeeding line of thought in physics enriched and supplemented, but never supplanted, those which had gone before; that the philosophical applications were in general superficial and ephemeral, the embodiment not of fundamental truth but of the

The nineteenth Josiah Willard Gibbs lecture delivered at Chicago, Illinois, November 23, 1945, under the auspices of the American Mathematical Society and the Mathematical Association of America: received by the editors December 6, 1945. 
prejudices of the men of the time; and that the impact on mathematics stimulated some of the most vital and valuable developments of that science.

I should like this evening to call to your attention the guiding principle which, more than any other, is running through the theoretical physics of the present, and to suggest that, though the mathematicians are well aware of it, they could nevertheless profit by following it even more closely. This is the wave equation. I shall not try to draw any philosophical implications from the fact that more and more parts of physics are now being explained in terms of the wave equation. I do not believe it has any philosophical implications at all. We are merely in that stage of the great development of physical thought in which we are exploring and exploiting the wave equation and its uses, as we have in the past explored and exploited Newtonian mechanics, the variational principle, statistics, tensor analysis, and many other lines. It will fit into a continually developing mosaic, enriching it and making it more powerful. We should be as wrong to base our philosophy on the principle of uncertainty and the wave mechanics as our ancestors were in basing theirs on Newtonian determinism; each is only a partial phase of the broader physical theory which is gradually unfolding itself. But mathematics may well be stimulated by studying the efforts of physicists to handle problems in the wave equation which are beyond the range of our present mathematical techniques, but not beyond the problems whose solution is not only useful but pressing.

The present importance of the wave equation is the culmination of a century and a half of continually increasing emphasis on partial differential equations. Laplace, Poisson, Fourier, Bernoulli, and many others, discovered the extraordinary similarity of the mathematical theory underlying the propagation of waves in strings and membranes and fluids and solids, the flow of heat and electricity and fluids, and the behavior of the gravitational and electrostatic potentials. These problems all have a mathematical framework in common. In each one there is a single dependent variable, the displacement or velocity, the temperature, the potential, and so on, and four independent variables, the three coordinates of space, and the one of time. The formulation of their fundamental laws in terms of differential equations leads us then at once to partial differential equations, relations between the derivatives of the dependent variable, which for the sake of illustration we may call $u$, with respect to the four independent variables $x, y, z$, and $t$. The remarkable feature of the problem is not this mathematical framework, but the fact that in every 
one of them the dependence on $x, y$, and $z$ comes through the Laplacian expression

$$
\frac{\partial^{2} u}{\partial x^{2}}+\frac{\partial^{2} u}{\partial y^{2}}+\frac{\partial^{2} u}{\partial z^{2}}
$$

which we may write as $\nabla^{2} u$, using the notation of Gibbs, in whose honor we are meeting this evening, and who more than any other clarified the relations between these branches of physics and vector analysis. The Laplacian appears in different ways in different problems. The form which we are particularly interested in tonight is the wave equation

$$
\nabla^{2} u-\frac{1}{v^{2}} \frac{\partial^{2} u}{\partial t^{2}}=0
$$

in which $v$ is a constant, which proves to be the velocity of propagation of the wave. But we have as well Laplace's equation, $\nabla^{2} u=0$, Poisson's equation, $\nabla^{2} u=f(x, y, z)$, where $f$ is a function of $x, y, z$, and the heat flow or diffusion equation, $\nabla^{2} u=a \partial u / \partial t$, where $a$ is a constant. These can be handled, as far as their time variation is concerned, in a common manner: we assume that $u$ varies with time according to the exponential function $e^{i p t}$, so that $u=w(x, y, z) e^{i p t}$. Thus the wave equation becomes $\nabla^{2} w+\left(p^{2} / v^{2}\right) w=0$, Laplace's equation is $\nabla^{2} w=0$, Poisson's equation is $\nabla^{2} w=f$, and the heat flow equation is $\nabla^{2} w=a i p w$. All of these can be considered as special cases of the general equation

$$
\nabla^{2} w+k^{2} w=f(x, y, z)
$$

which we may call the inhomogeneous wave equation. By setting $k=0$, or $f=0$, we can obtain our various special cases.

We have here a partial differential equation for a function $w(x, y, z)$, whose solution is one of the classical problems of mathematical physics. Two broad approaches to its solution have been made, the method of expansion in orthogonal functions, and of Green's functions. Both are based first on a consideration of the related homogeneous equation $\nabla^{2} w+k^{2} w=0$. It is perfectly simple to obtain solutions, in fact an indefinitely large assortment of solutions, of this equation. The trick is to find solutions satisfying certain desired boundary conditions. If we desire solutions of the equation, for instance within a closed volume, satisfying the condition that $w=0$ over the surface of the volume, then we can show, if $k^{2}$ is positive, that solutions are possible only for certain discrete values of $k$, the characteristic values or proper values or eigenvalues, which we may denote by a subscript, as $k_{n}$; the corresponding functions $w_{n}$, the char- 
acteristic functions or proper functions or eigenfunctions, may then easily be shown to possess a property of orthogonality,

$$
\int w_{n} w_{m} d v=0 \quad \text { if } n \neq m
$$

where the integral is extended over the volume. The general solution of our homogeneous wave equation is then shown to be expressible as a sum of particular solutions,

$$
u=\sum_{n} A_{n} w_{n}(x, y, z) e^{i k_{n} v t}
$$

where the $A_{n}$ 's are arbitrary, and may be used to satisfy initial conditions at $t=0$. To satisfy these initial conditions, we must expand the initial value of $u$, say $W(x, y, z)$, in series of the form

$$
W(x, y, z)=\sum_{n} A_{n} w_{n}
$$

and so determine the constants $A_{n}$. Such an expansion is easily carried out, in virtue of the orthogonality of the $w_{n}$ 's: we merely multiply both sides of the equation by a particular $w_{m}$, integrate over the volume, and have

$$
A_{m}=\int W w_{m} d v / \int w_{m}^{2} d v
$$

This is an expansion analogous to a Fourier expansion, and many of the properties characteristic of the Fourier expansion hold here too.

We may set up a space of an infinite number of dimensions in which the $A_{m}$ 's are plotted as the coordinates of a point. Such a point represents a function; the space is called a function space, or a Hilbert space, and the property of orthogonality can be interpreted geometrically in function space. Through such paths we are led to a sort of vector analysis in a space of an infinite number of dimensions; that is, we are led to a form of linear algebraic equations formally equivalent to the solution of the partial differential equation. This is a transformation of great power in all problems of this type, a transformation which for example allows us to solve problems in perturbations: if we know the solution of a problem near to the desired one, we can expand the solution of the desired problem in terms of the characteristic functions of the similar soluble problem, and, using the algebraic form of the problem, we can find the expansion coefficients as power series in the small perturbation.

By using the method of orthogonal expansion, we can solve the in- 
homogeneous wave equation, for we can expand both $w$ and the inhomogeneous function $f(x, y, z)$ in series in the $w_{n}$ 's, and equate terms on both sides of the equation. An interesting case is found where the inhomogeneous part of the equation varies sinusoidally with time. If, for instance, we have the equation

$$
\nabla^{2} u-\frac{1}{v^{2}} \frac{\partial^{2} u}{\partial t^{2}}=f(x, y, z) e^{i \omega t}
$$

and if we let $u=\sum_{n} U_{n} w_{n} e^{i \omega t}, f=\sum_{n} F_{n} w_{n}$, then, remembering that $\nabla^{2} w_{n}+\left(\omega_{n}^{2} / v^{2}\right) w_{n}=0$ (where $\omega_{n}^{2} / v^{2}$ is written for the earlier $k_{n}^{2}$ ), we have

$$
\sum_{n} U_{n}\left(\frac{\omega^{2}-\omega_{n}^{2}}{v^{2}}\right) w_{n}=\sum_{n} F_{n} w_{n}
$$

or, equating terms,

$$
U_{n}=F_{n} v^{2} /\left(\omega^{2}-\omega_{n}^{2}\right),
$$

showing that the coefficients $U_{n}$ show a dependence on $\omega$ similar to that of the amplitude of a linear oscillator of natural frequency $\omega_{n}$, forced by an external frequency $\omega$. We are led by following this argument to a theory of the normal modes of oscillation, closely related to the normal coordinates of a vibrating system of a finite number of degrees of freedom. We find, in fact, that we can build up the whole theory of the orthogonal functions and normal modes of the wave equation by a generalization of the theory of mechanical oscillations of a system of a finite number of degrees of freedom, to the case where the number of degrees of freedom becomes infinite.

The extensive development of the theory of orthogonal functions, which we have merely sketched, is very beautiful and very general, but its usefulness is severely limited by the very narrow range of problems for which we can actually obtain the solutions and find the orthogonal functions. The problems which are soluble by classical means are only those in which a separation of variables is possible; that is, in which $w$ can be written as a product of functions $X(\xi) H(\eta) Z(\zeta)$, where $\xi, \eta, \zeta$ are three variables, functions of $x, y, z$, such that the boundaries are determined by setting either $\xi, \eta$, or $\zeta$ equal to constants. It can be shown that there are only eleven sets of coordinates in which separation can be effected, the familiar ones being rectangular, cylindrical, spherical, and ellipsoidal coordinates. These soluble problems are important, but the insoluble ones, with more complicated boundary conditions, are more so, and at present 
we know no elegant ways of getting solutions in such cases. Physicists attempt to devise approximate ways of computing the characteristic functions and characteristic numbers of such problems, but these methods lack generality. No problem which the mathematician could attack would be more useful to the physicist at present than a real advance in the solution of the non-separable wave equation.

The other method of solving the wave equation, which shows as much generality as the method of orthogonal functions, is Green's method. The most familiar example of this is found in the solution of Poisson's equation, $\nabla^{2} \phi=-4 \pi \rho(x, y, z)$, for the potential $\phi(x, y, z)$ determined by a density of charge $\rho(x, y, z)$. The solution is $\phi=\int(\rho / r) d v$, where $r$ is the distance from the point where $\rho$ is computed to the place where we are finding $\phi$. This holds if the boundary condition is that the potential is to vanish at infinite distance. If instead the potential is to have specified values over a finite boundary, surface integrals must be added to the volume integral, arising from the socalled Green's distribution over the surface. If we have the wave equation instead of Poisson's equation, we then meet the Kirchhoff solution, in which we must use the retarded potential, computing the $\rho$ which appears in the integral not at the time $t$ at which we want to find $\phi$, but at a time earlier by the amount $r / v$, such that a disturbance leaving the point of integration at the earlier time, traveling with the velocity $v$, would reach the point $x y z$ at time $t$. These solutions are very general and very powerful, but often not very convenient for computation. We can naturally show the equivalence of the method of orthogonal functions and of Green's function, and in this way can exhibit interesting interrelationships between the theory of integral equations (to which Green's method naturally leads us) and the theory of orthogonal functions and of function space. The mathematical interrelationships of all these ways of handling the wave equation should be the familiar stock in trade of every physicist. Unfortunately they are not; for the conventional mathematical texts and college and university courses concentrate almost entirely on the soluble cases of potential theory rather than on the powerful general methods and the relationships between them. The physicist, rather than the mathematician, is left with the main burden of expanding this branch of mathematics.

Now let us inquire why and how these methods of handling the wave equation have come to have so much more importance in the last few years. This has come about largely through the wave mechanics. Everyone realizes by now that it has been found that the correct formulation of mechanical problems is through Schrödinger's 
equation, an equation which is essentially like the wave equation, only with a velocity which is a function of $x, y, z$. Such an equation can perfectly well occur in other branches of physics, for instance in optics, if the index of refraction varies from point to point, but it had been largely neglected by the mathematicians. Schrödinger's equation, however, has forced the physicist to hunt for ways of solving this case, approximately or exactly. The problem is further complicated by the fact that there are not merely the three coordinates $x, y, z$ in addition to time, but $3 n$ coordinates, where $n$ is the number of particles concerned in the problem. In spite of this great added difficulty, great progress has been made in discussing the practically important cases of Schrödinger's equation. The general method of orthogonal functions, with all its ramifications, still applies. Green's method, on the other hand, has had almost no study as related to Schrödinger's equation; maybe there are unsuspected advances to be made in that direction. At any rate, wave mechanics has widened the horizons and strengthened the techniques of the physicist when dealing with the wave equation. It has also clearly shown that the importance of the method of variations, and the method of classical mechanics, which I mentioned earlier, was exaggerated in earlier periods. For it shows that the principle of least action is an approximate principle giving the path of a ray, as Fermat's principle is in optics, and is merely a derived result of the wave equation; and that classical mechanics is likewise a limiting case, describing the limiting motion of a so-called wave packet, in the case of large-scale objects.

Wave mechanics has greatly broadened and strengthened the grasp of the physicist on the wave equation, and has made him more anxious than ever to learn more and more powerful techniques for handling it. This broadened grasp has reacted in other applications of physics. My colleagues Morse and Bolt have been applying the methods learned in wave mechanics to the study of acoustics, to sound waves in rooms and other enclosures, with the result that they are changing the whole line of development of that science. Many of us working on radar during the war have been applying our knowledge of wave mechanics to help us in the solution of electromagnetic problems, with very satisfactory results. For instance, I have been using the method of expansion in orthogonal functions for a general study of electromagnetic oscillations in a cavity, leading to formulas like the ones I mentioned earlier for the solution of an inhomogeneous wave equation, which resembles the formula for a forced oscillator. In electromagnetic language, this becomes the formulation of the behavior of an oscillating cavity in terms of an equivalent circuit, and it gives 
a complete theory of the circuit-like behavior of a cavity, a result of great practical importance in microwave theory. This is an example of a case where the general theory has important uses, even in the absence of exact solutions for special cases; for the general theory tells us the type of functional behavior we can expect, in terms of certain undetermined constants, and in our practical work we have determined those constants experimentally, in cases where we could only estimate them crudely from numerical calculation.

The applications of wave theory will extend far beyond such examples, however. For it becomes clear that wave mechanics is furnishing at least a beginning for the study of nuclear forces, and the behavior of particles of very high energies. We are then at the threshold of a great new field of theoretical physics, for which we are very conscious that we do not at present possess the answer. Such theory as we have is a development of the types of theory that I have sketched this evening. On the other hand, it is very tentative and unsatisfactory. For one thing, there are points of the utmost importance in which the theory, which takes the form of an expansion in power series, gives series that fail to converge. In some cases, these divergences seem to result from an unhappy choice of the form of expansion to use; in other cases they are inherent. In those cases, more than in any other which physicists have yet had to face, convergence or divergence is a major problem. Here it is the physicists who are likely to elucidate the inner nature of the problem, as Gibbs, in a very similar case, pointed out the true nature of the so-called Gibbs phenomenon in Fourier expansion. It seems likely that the real solution of our physical problems will not be accomplished without at the same time bringing about real extensions of our mathematical knowledge.

At this point, I return to my introduction. More and more of physics is being found to be subject to the wave equation. The physicist needs to know more than he does about it, both in the matter of the general theory, and of the solution of special problems. Yet the physicist finds very little help from the mathematician. For every mathematician like von Neumann who realizes these problems, and contributes practically to them, there are twenty who seem to have no interest in them, who either work in fields of only remote interest to physics, or who stress the older and more familiar parts of mathematical physics. Is it any wonder that in such a situation the physicist, looking at the mathematicians, feels that they have strayed from the path which has led to the past greatness of mathematics, and feels that they will not regain this path until they again resolutely enter the main current of progress of mathematical physics, the cur- 
rent which in the past has led to the most fruitful development of mathematics? Gibbs was great because he kept close to earth, always knew what his theory was for. That, the physicist firmly feels, is the only path through which the mathematician of the present can achieve greatness.

Massachusetts Institute of Technology 